Manzanilla-Quiñones et al.

\title{
Escenarios climáticos (CMIP-5) para la Reserva de la Biosfera Pantanos de Centla, Tabasco, México
}

\section{Climate scenarios (CMIP-5) for the Reserva de la Biosfera Pantanos de Centla, Tabasco, Mexico}

Ulises Manzanilla-Quiñones $3:$ [D Gilberto Pozo-Montuy ${ }^{2}$ (i)

Patricia Delgado-Valerio [D Aldo Rafael Martinez-Sifuentes ${ }^{3}[0$ Oscar Alberto Aguirre-Calderón $n^{4}$ 으

${ }^{1}$ Universidad Michoacana de San Nicolás de Hidalgo, Facultad de Agrobiología "Presidente Juárez", Avenida Revolución esquina con Berlín s/n Colonia Viveros, CP. 60170, Uruapan, Michoacán, México.

${ }^{2}$ Academia de ingeniería Ambiental, TecNM. Campus de los Ríos. Km 3 Carretera Balancán-Villahermosa S/N, CP. 86930. Balancán, Tabasco, México.

${ }^{3}$ Instituto Nacional de Investigaciones Forestales, Agrícolas y Pecuarias. Centro Nacional de Investigación Disciplinaria en Relación Agua, Suelo, Planta, Atmósfera, Km. 6.5 margen derecha Canal de Sacramento, CP. 35140, Gómez Palacio, Durango, México.

${ }^{4}$ Universidad Autónoma de Nuevo León, Facultad de Ciencias Forestales, Carretera Nacional km 145, CP. 67700, Linares, Nuevo León, México.

*Autor de correspondencia: ulises_manza@hotmail.com

Artículo científico

Recibido: 02 de junio 2020 Aceptado: 04 de noviembre 2020

Como citar: Manzanilla-Quiñones U, Pozo-Montuy G, Delgado-Valerio P, Martínez-Sifuentes AR, AguirreCalderón OA (2021) Escenarios climáticos (CMIP-5) para la Reserva de la Biosfera Pantanos de Centla, Tabasco, México. Ecosistemas y Recursos Agropecuarios Núm. Esp. I: e2588. DOI: 10.19136/era.a8nl.2588
RESUMEN. El presente estudio utilizó datos de precipitación y temperatura de 1960-2015 y de Modelos de Circulación Global (MCG) CNRMCM5 y HADGEM2_ES, proyectados a 2045-2069 y 2075-2099; bajo dos rutas de concentraciones de emisiones de gases de efecto invernadero (RCP); 4.5 (constantes) y 8.5 (altas). El objetivo fue probar la hipótesis de que la RBPC se convertiría en un sitio altamente expuesto al aumento en temperatura promedio anual e inundable por el incremento del nivel del mar. Los registros de precipitación y temperatura se descargaron de seis estaciones meteorológicas y capas climáticas de MCG para 2045-2069 y 2075-2099 (RCP 4.5 y 8.5) a una resolución de $1 \mathrm{~km}^{2}$. Las pruebas estadísticas utilizadas en los datos permitieron detectar tendencias de aumento $(p<0.05)$ a través del tiempo. Debido a la incertidumbre de los escenarios climáticos a futuro, se probaron y analizaron cuatro escenarios. Los resultados indican una tendencia de aumento significativo en temperatura promedio anual de 0.34 a $0.82{ }^{\circ} \mathrm{C}$ para $1960-2015$. Los escenarios climáticos futuros proyectaron que la RBPC sería más cálida, seca e inundada por el incremento del nivel del mar de 13 a $33 \mathrm{~cm}$, lo que propiciará en la formación de un delta costero, abarcando entre 49.3 a $49.5 \%$ de la RBPC durante 2045-2099. Conforme con los análisis climáticos, la RBPC podría ser un área altamente expuesta al calentamiento global e incremento del nivel del mar. Este último ocasionaría cambios biogeoquímicos importantes en los cuerpos de agua dulce del área.

Palabras clave: Análisis espacial, área natural protegida, aumento en temperatura, cambio climático, incremento del nivel del mar.

ABSTRACT. The present study used precipitation and temperature data between 1960-2015 and Global Circulation Models (GCM) CNRMCM5 and HADGEM2_ES, projected from 2045-2069 and 2075-2099; under two paths of concentrations of greenhouse gas emissions (RCP); 4.5 (constant) and 8.5 (high). The objective was test the hypothesis that the RBPC would become a site highly exposed to the effects of annual temperature average increase and flooding due to sea-level increase. Precipitation and temperature records were downloaded from six meteorological stations and the climatic layers from GMC for 2045-2069 and 2075-2099 (RCP 4.5 and 8.5) at a resolution of $1 \mathrm{~km}^{2}$. The statistical tests used in the data allowed to detect increase trends $(p<0.05)$ through time. Due to the uncertainty of future climate scenarios, four GCM were tested and analyzed. The results indicate a significant increase trend in annual temperature average from 0.34 to $0.82{ }^{\circ} \mathrm{C}$ for $1960-2015$. The climate scenarios projected that the RBPC would be warmer, drier, and flooded by a sea-level increase from 13 to $33 \mathrm{~cm}$, which would lead to the development of a coastal delta, covering 49.3 to $49.5 \%$ of the RBPC during 2045-2099. According to the climatic analyses, the RBPC would show a high climatic exposure in its biological and ecological systems. According to climate analyses, the RBPC would be a highly exposed area to the effects of climate change and sea-level increase. The latter would affect important biogeochemical changes to the freshwater bodies of the area.

Key words: Climate change, protected natural area, sea level rise, spatial analysis, temperature increase. 


\section{INTRODUCCIÓN}

El cambio climático es un fenómeno global bien documentado y estudiado por diversas instituciones, principalmente por el Panel Intergubernamental de Expertos Sobre el Cambio Climático (IPCC 2014) y por dependencias nacionales como el Instituto $\mathrm{Na}$ cional de Ecología y Cambio Climático (INECC), Unidad de Información para las Ciencias Atmosféricas y Ambientales (UNIATMOS) de la Universidad Nacional Autónoma de México (UNAM) y la Comisión Nacional de Áreas Naturales Protegidas (CONANP) en México. En 2013 el Panel Intergubernamental de Cambio Climático (IPCC 2013) declaró que el calentamiento global observado durante la mitad del siglo pasado fue producto de acciones antropogénicas, lo que sumado a la variabilidad climática ocurrida entre el periodo 1901 a 2012 evidenciaron un aumento significativo de $1{ }^{\circ} \mathrm{C}$ en la temperatura promedio global actual. Hacia futuro (2050) se proyectan aumentos significativos de hasta $2{ }^{\circ} \mathrm{C}$ en la temperatura promedio mundial (IPCC 2014); mientras que en México los escenarios climáticos estiman un aumento en temperatura promedio anual de $2.3{ }^{\circ} \mathrm{C}$ (2050) y $3.7^{\circ} \mathrm{C}$ (2090) (Sáenz-Romero et al. 2010).

Un escenario de cambio climático es la diferencia entre el clima actual de una región geográfica y una proyección climática a futuro del mismo sitio (Magaña et al. 2012, Fernández-Eguiarte et al. 2015). Estos no son simples pronósticos climáticos, ya que cada escenario es una posible ruta alternativa de comportamiento del clima a futuro (FernándezEguiarte et al. 2015, Manzanilla et al. 2018) y son útiles en los estudios de evaluación y estimación de los efectos del cambio climático a futuro, donde el principal recurso empleado son los Modelos de Circulación Global (MCG), los cuales son una representación numérica tridimensional de la dinámica atmosférica y de la circulación global alrededor de la tierra (Jáuregui 2003, FernándezEguiarte et al. 2015). En ellos se simulan procesos físicos de la atmósfera, océanos y la superficie terrestre, además constituyen una herramienta muy útil en las proyecciones de respuesta del clima a futuro bajo diversos escenarios hipotéticos de au- mento en las concentraciones de los gases efecto invernadero en la atmósfera (IPCC 2013, FernándezEguiarte et al. 2015).

Debido a los efectos del cambio climático se prevé que durante el presente siglo la estructura y función de los ecosistemas terrestres y acuáticos a escala global, regional y local se modifiquen de manera considerable (Gutiérrez y Trejo 2014, IPCC 2014, Sáenz-Romero et al. 2015, ManzanillaQuiñones et al. 2019). Las áreas costeras son ecosistemas altamente vulnerables a los efectos del cambio climático mundial (IPCC 2014, Miranzo y del Río 2015, Ramos et al. 2016). Estas representan la primera porción terrestre que afrontará los eventos derivados del cambio climático como huracanes, inundaciones y el aumento del nivel del mar (IPCC 2014, CONANP y PNUD México 2019). EI incremento del nivel del mar afectará a las zonas costeras de México, siendo la franja costera del Golfo de México y el mar Caribe las más vulnerables al calentamiento global (SEMARNAP 1997). Estudios realizados sobre la línea costera de Cuba estiman un aumento del nivel del mar de $27 \mathrm{~cm}$ para 2050 y de 85 $\mathrm{cm}$ hacia finales de siglo (CITMA 2012), mientras que en el Golfo de México, específicamente el complejo de áreas naturales protegidas (ANPs) Reserva de la Biosfera Pantanos de Centla-Área de Protección de Flora y Fauna Laguna de Términos se prevén incrementos en el nivel del mar de $13 \mathrm{~cm}$ durante 2050 y de $33 \mathrm{~cm}$ a finales de siglo (CONANP y PNUD México 2019).

La Reserva de la Biosfera Pantanos de Centla (RBPC) es una ANP de alta relevancia biológica y ecológica del sureste de México que forma parte del Corredor Biológico Mesoamericano (Álvarez 2013). La flora de la RBPC está representada por 813 taxones de plantas vasculares, distribuidas en 462 géneros y 115 familias, de las cuales 731 son especies nativas, 64 introducidas y 18 cultivadas (López-Jiménez et al. 2020). En cuanto a la fauna, se tienen registros de 674 especies; 172 peces, 27 anfibios, 68 reptiles, 104 mamíferos, 255 aves, 35 moluscos y 13 crustáceos (Velasco et al. 2005, SoriaBarreto et al. 2018, Trinidad-Ocaña et al. 2018), de las cuales el Manatí (Trichechus manatus), el Jaguar 
(Panthera onca) y los monos aulladores (Alouatta pigra y $A$. palliata) se consideran especies prioritarias en la conservación (CONANP y PNUD México 2019). En un contexto ecológico la RBPC posee comunidades vegetales de tipo manglar, dunas costeras, vegetación acuática y subacuática (Vega-Moro 2005), que fungen como sitios de regulación contra inundaciones, brindan refugio a poblaciones de aves y sirven de áreas de crianza y de alimentación de peces de valor comercial (Barba 2005).

Pese a la riqueza biológica y ecológica que posee el ANP, los estudios sobre su variabilidad climática y de escenarios de cambio climático son incipientes y de carácter prioritario en la evaluación de los efectos del cambio climático a nivel regional (CONANP y PNUD México 2019). Lo cual es sumamente relevante en el manejo y conservación de los recursos naturales, específicamente en humedales y ecosistemas costeros, ya que ambos serían de los primeros biotas en experimentar los cambios en el clima a futuro (IPCC 2014). En el presente estudio se utilizaron datos de precipitación y temperatura actuales (1960-2015) de estaciones meteorológicas y capas climáticas de Modelos de Circulación Global (MCG) proyectados a futuro (20452069 y 2075-2099) para probar la hipótesis de que la RBPC se convertiría en un sitio altamente expuesto a los efectos del cambio climático (aumento en temperatura promedio anual y ascenso en el nivel del mar) a futuro. Por lo que para probar dicha hipótesis se formularon los siguientes objetivos específicos: a) Analizar las series de tiempo anuales de precipitación acumulada y temperatura promedio de las estaciones meteorológicas cercanas a la RBPC, b) Generar capas ráster de precipitación y temperatura, c) Calcular las diferencias en precipitación y temperatura entre el periodo actual (1960-2015) y futuro (2045-2069 y 2075-2099) de los MCG CNRMCM5 y HADGEM2_ES, bajo dos trayectorias de emisiones de gases de efecto invernadero (RCP) de 4.5 (constantes) y 8.5 (altas) y d) Estimar los niveles de afectación del cambio climático en la RBPC para 2045-2069 y 2075-2099.

\section{MATERIALES Y MÉTODOS}

Área de estudio. La RBPC es un área natural protegida que se ubica entre las coordenadas geográficas $17^{\circ} 57^{\prime} 53^{\prime \prime}$ y $18^{\circ} 39^{\prime} 03^{\prime \prime}$ LN y $92^{\circ} 06^{\prime} 39^{\prime \prime}$ y $92^{\circ} 47^{\prime} 58^{\prime \prime}$ LO (INCEC 2000); con una superficie de 302706.62 ha (Figura 1).

Clima. De acuerdo con la clasificación climática de Köppen, modificada por García (1998), el área presenta dos tipos de climas dominantes; Am(f) cálido húmedo, con temperatura promedio anual mayor de $22{ }^{\circ} \mathrm{C}$ y temperatura del mes más frío mayor a $18^{\circ} \mathrm{C}$ y Aw2( $\left.x^{\prime}\right)$ cálido subhúmedo, con temperatura promedio anual mayor de $22^{\circ} \mathrm{C}$ y temperatura del mes más frío mayor de $18{ }^{\circ} \mathrm{C}$, siendo el primero el que mayor cantidad de humedad relativa presenta.

Uso del suelo y vegetación. De acuerdo con los datos de la serie VI uso del suelo y vegetación del INEGI (2016), la vegetación dominante en el ANP corresponde al tipo tular, seguida de popal, manglar, pastizales inducidos y remanentes de selvas altas perennifolias y bajas.

Edafología y topografía. Los suelos dominantes son del tipo Gleysol éutrico $(\mathrm{Ge})$ y Gleysol vertico (Gv) (INEGI 2014). El gradiente altitudinal dentro de la RBPC es a nivel de mar, con elevación máxima de $30 \mathrm{~m}$, situada en el centro-este de la Reserva. La pendiente es escasa en más del $95 \%$ de la RBPC (INEGI 2020).

Hidrología. La RBPC se encuentra localizada en la región hidrológica Grijalva-Usumacinta y abarca tres cuencas: Usumacinta al norte-centro del ANP; Laguna de Términos, al este y Rio Grijalva, al sur-oeste de la Reserva, siendo esta última la más extensa. Los ríos más importantes dentro del ANP son el Grijalva y el Usumacinta, este último, es considerado el más caudaloso de México (INEC 2000, Barba et al. 2006). La RBPC cuenta con aproximadamente 110 ecosistemas lénticos, la mayoría concentrados en las zonas centro y sur y cubren el $44 \%$ del ANP (Barba et al. 2006). 


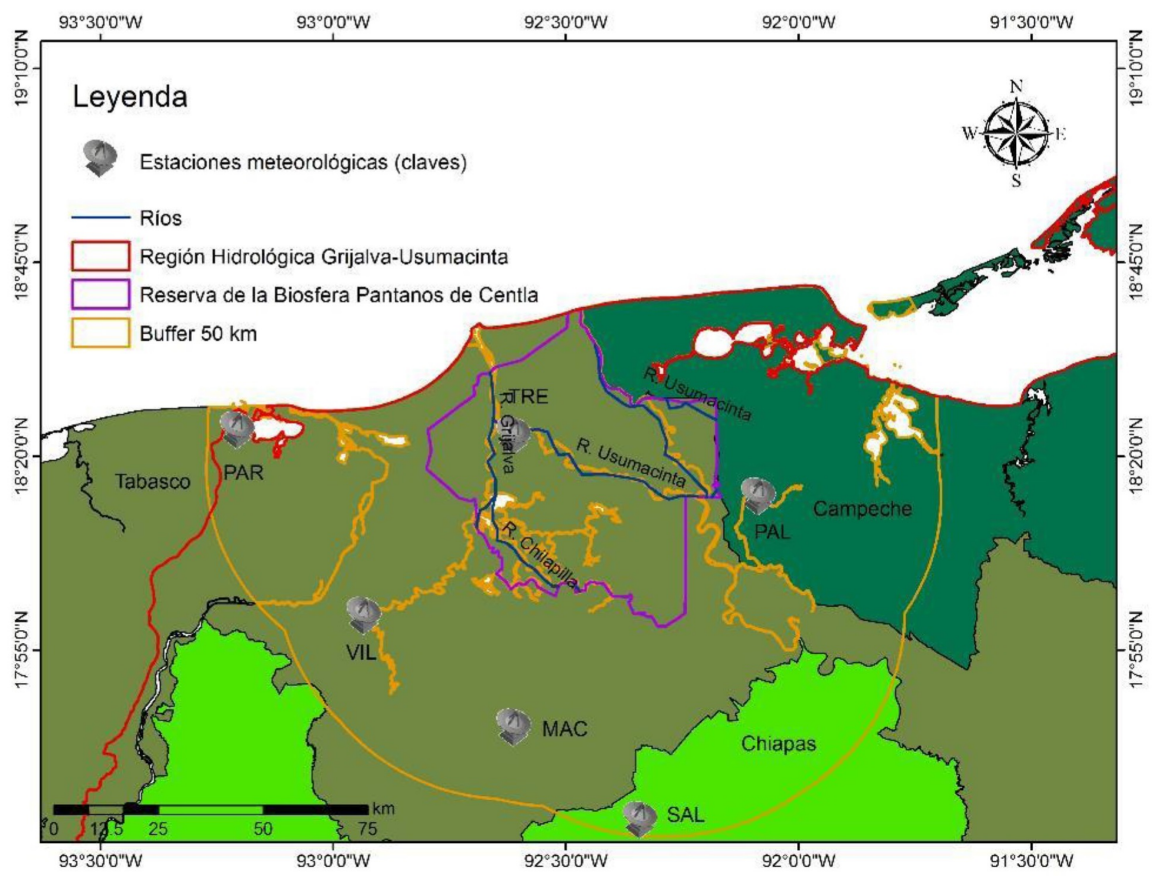

Figura 1. Localización geográfica de la RBPC y ubicación de estaciones meteorológicas cercanas al ANP.

Datos meteorológicos. Los registros de precipitación y temperatura de las estaciones meteorológicas se obtuvieron a partir de la delimitación de un Buffer o área de influencia de $50 \mathrm{~km}$ de distancia lineal con referencia al límite poligonal de la RBPC (Figura 1). Se descargaron las bases de datos climáticos con registros mayores a 30 años de actividad meteorológica de la plataforma CLICOM (CLICOM 2020) de 16 estaciones; 11 distribuidas en Tabasco, tres en Chiapas y dos en Campeche.

Análisis y depuración de datos. Debido a las múltiples inconsistencias en los registros meteorológicos, se aplicaron varios filtros de control de calidad de datos. Primero se descartaron los registros de las estaciones meteorológicas con más de $10 \%$ de los datos incompletos. Segundo se definió un periodo de actividad climática común (Manzanilla-Quiñones et al. 2020), el cual presentó más del $95 \%$ de los datos completos entre estaciones, y tercero, los datos faltantes de precipitación y temperatura se estimaron mediante la aplicación de medias móviles de orden $n=5$ (Schulz 1976). Al final del proceso de depu- ración de los datos se obtuvieron las bases de seis estaciones meteorológicas que cumplieron con los filtros de calidad establecidos (cuatro en Tabasco, una en Chiapas y una en Campeche). Las cuales cuentan con los registros mensuales completos de precipitación acumulada y temperatura promedio del periodo 1960-2015 (Tabla 1).

Análisis de series de tiempo y de tendencias. Las series de tiempo se emplearon para detectar la variabilidad climática en los datos meteorológicos, la cual puede ser clasificada como estacional o no estacional (McCuen y Snyder 1986). Las series de tiempo se realizan con el fin de identificar las posibles tendencias (cambios en la varianza a través del tiempo) en las series climáticas analizadas y se corroboraron mediante la aplicación de pruebas de tendencias. Los análisis se efectuaron con los datos completos de temperatura promedio y precipitación acumulada anual de las estaciones meteorológicas seleccionadas. A las series temporales anuales de precipitación y temperatura promedio se les aplicó la prueba de homogeneidad de varianzas de Bartlett 
Tabla 1. Descripción de las seis estaciones meteorológicas cercanas $(<50 \mathrm{~km})$ a la RBPC.

\begin{tabular}{llllll}
\hline Estado & Estación & Clave & $\begin{array}{l}\text { Distancia lineal } \\
\text { al ANP }(\mathrm{km})\end{array}$ & $\begin{array}{l}\text { Promedio anual PPT } \\
1960-2015(\mathrm{~mm})\end{array}$ & $\begin{array}{l}\text { Promedio anual TMED } \\
1960-2015\left({ }^{\circ} \mathrm{C}\right)\end{array}$ \\
\hline Tabasco & Macuspana & MAC & 32.12 & 2390.16 & 26.94 \\
& Paraíso & PAR & 42.96 & 1780.48 & 26.58 \\
& Tres Brazos & TRE & $0^{*}$ & 1697.37 & 26.68 \\
& Villahermosa & VIL & 31.32 & 1972.90 & 27.20 \\
Chiapas & Salto de Agua & SAL & 45.51 & 3308.19 & 26.47 \\
Campeche & Palizada & PAL & 8.77 & 1881.66 & 26.86 \\
\hline $0^{*}$ la estación se encuentra dentro de la RBPC.
\end{tabular}

(Bartlett 1937), mientras que los de tendencias se efectuaron mediante la aplicación de la prueba de Mann-Kendall (Kendall 1938); ambos análisis se realizaron con el programa $\mathrm{R}$ versión 4.0 (R Core Team 2020).

Generación de capas climáticas. Posteriormente los datos climáticos promedio del periodo 1960-2015 se utilizaron para generar las capas ráster de precipitación acumulada anual y temperatura promedio anual mediante interpolaciones por distancia inversa ponderada (IDW) en el programa QGIS versión 3.2 (QGIS 2018) a resolución espacial de $90 \mathrm{~m}$. Las capas climáticas ráster fueron recortadas al tamaño del ANP con la herramienta clip del programa QGIS versión 3.2 (QGIS 2018), lo que permitió visualizar la distribución de la variación histórica de la precipitación anual acumulada y de la temperatura promedio anual de 1960-2015 en la RBPC.

Índices de sequías. La severidad de las sequías en la RBPC, se determinó por el Índice de Severidad de Sequías de Palmer (PDSI) mediante el uso de los datos espaciales mensuales de la plataforma Mexican Drought Atlas (Stahle et al. 2016) de febrero-abril de 1960-2012 (datos disponibles). La aplicación del índice ayudó a determinar el impacto de las sequías en la RBPC.

Modelos de Circulación Global. Las 12 capas ráster mensuales de precipitación acumulada y temperatura promedio utilizadas se descargaron de los Modelos de Circulación Global (MCG) CNRMCM5 del Centro Nacional de Investigaciones Meteorológicas de Francia y HADGEM2_ES de la Oficina de Meteo- rología del Centro Hadley de Reino Unido. Ambos modelos fueron proyectados a 2045-2069 (futuro medio) y 2075-2099 (futuro lejano) bajo dos escenarios hipotéticos de emisiones de gases de efecto invernadero; de emisiones constantes (RCP 4.5) y de emisiones altas (RCP 8.5). Los datos de estos modelos fueron obtenidos de la plataforma UNIATMOS de la UNAM, a una resolución espacial de $1 \mathrm{~km}^{2}$ y en formato Tif (Fernández-Eguiarte et al. 2015) y generados a partir de Modelos Regionales del Proyecto CMIP-5 (Proyecto de Intercomparación de Modelos Acoplados Fase 5) del Panel Intergubernamental de Expertos Sobre el Cambio Climático (IPCC). Además son de los MCG más empleados en la generación de escenarios climáticos a futuro en México (Fernández-Eguiarte et al. 2015, Manzanilla y Aguirre 2017, Manzanilla-Quiñones et al. 2019).

Clima a futuro. Debido al inconveniente entre escalas espaciales (actuales y futuras) fue necesario reajustar la resolución espacial $\left(1 \mathrm{~km}^{2}\right)$ de las capas climáticas futuras de los MCG a una escala de $90 \mathrm{~m}$ mediante la aplicación de un remuestreo de tipo bilineal en el programa ArcGis versión 10.3 (ESRI 2014) esto se realizó con el objetivo de mejorar las estimaciones en las superficies climáticas. Posteriormente, la precipitación acumulada anual y la temperatura promedio anual a futuro se calcularon con el módulo algebra de mapas del programa ArcGis versión 10.3 (ESRI 2014). Para después recortar al tamaño del ANP con la herramienta clip del programa QGIS versión 3.2. (QGIS 2018). Este ajuste permitió estimar la precipitación acumulada anual y la temperatura promedio anual de la RBPC de 20452069 y $2075-2099$. 
Generación de escenarios climáticos. La estimación de los escenarios climáticos de cada horizonte y forzamiento radiactivo (RCP) de 4.5 y 8.5 se realizaron en el módulo algebra de mapas del programa ArcGis versión 10.3, donde se ingresaron las capas climáticas actuales (1960-2015) y futuras (2045-2069 y 2075-2099) ajustadas a una misma escala espacial, y se calcularon las diferencias entre ambas variables. El cálculo de las diferencias sirvió para generar los escenarios climáticos del ANP de 2045-2069 y 2075-2099. De acuerdo con los valores de incremento y disminución de cada variable analizada se clasificaron los escenarios climáticos en conservadores (valores mínimos) y extremos (valores máximos).

Afectación por cambio climático. El nivel de afectación por los efectos de cambio climático en la superficie de la RBPC se estimó con base en los valores de aumento (no deseados) de la temperatura promedio anual (umbral térmico $\leq 2{ }^{\circ} \mathrm{C}$ de aumento en temperatura promedio anual mundial) reportados por el IPCC (2014) de cada horizonte de tiempo proyectado.

La inclusión de agua marina en la RBPC se calculó mediante transferencia de los valores de incremento en el nivel del mar reportados (CONANP y PNUD México 2019) en el modelo digital de elevación con resolución espacial de $15 \mathrm{~m}$ de la zona de estudio. La reclasificación de los valores de incremento en el nivel del mar permitió estimar la superficie que se vería afectada (inundada) por la entrada de agua marina a partir de 2045-2069. La edición final de los mapas climáticos y de afectación asociada con los efectos del cambio climático en la RBPC se realizó en un ambiente de SIG en el programa ArcGis versión 10.3 (ESRI 2014).

\section{RESULTADOS}

Análisis climático de estaciones meteorológicas. Los resultados de los análisis de series de tiempo de las seis estaciones meteorológicas cercanas a la RBPC indicaron una estacionalidad en precipitación (temporada de lluvias) que se presenta de mayo a noviembre y un periodo de sequías registrado de febrero a abril. Las pruebas de tendencias de precipitación anual no presentaron cambios significativos ( $p>0.05$ ) durante el periodo 1960-2015, lo que denotó una homogeneidad de varianzas constantes a través del tiempo en las seis estaciones evaluadas, y por lo tanto la ausencia de alteraciones climáticas en las series anuales analizadas (Mann-Kendall, $p$ > 0.05, Tabla 2).

Tabla 2. Resultados de la prueba de Mann-Kendall de las seis estaciones meteorológicas cercanas a la RBPC.

\begin{tabular}{lccc}
\hline Variable & Estación (clave) & Valor tau & Valor $\mathrm{p}$ \\
\hline Precipitación acumulada & MAC & 0.0714 & 0.4410 \\
anual & PAR & -0.0051 & 0.9605 \\
& TRE & -0.0377 & 0.6870 \\
& VIL & 0.0312 & 0.7397 \\
& SAL & -0.0039 & 0.9718 \\
& PAL & -0.1420 & 0.1251 \\
Temperatura promedio & MAC & 0.4470 & $0.0000^{* *}$ \\
anual & PAR & 0.2340 & $0.0111^{*}$ \\
& TRE & 0.4560 & $0.0000^{* *}$ \\
& VIL & 0.3360 & $0.0002^{* *}$ \\
& SAL & -0.1600 & 0.0833 \\
& PAL & 0.2010 & $0.0289^{*}$ \\
\hline
\end{tabular}

Tau: valores positivos indican una tendencia de aumento, mientras que los valores negativos señalan una tendencia de disminución. *significativo $(p<0.05) .{ }^{* *}$ significativo $(p<0.01)$.

En lo que se refiere a temperatura, no se encontró una estacionalidad en la temperatura promedio durante 1960-2015. Pero los análisis de homocedasticidad de varianzas de Bartlett señalaron comportamientos irregulares $(p>0.05)$, que revelaron la presencia de tendencias en las series anuales evaluadas. La prueba de Mann-Kendall detecto tendencias significativas $(p<0.05)$ en aumento de la temperatura promedio anual de 1960-2015 en cinco de las seis estaciones meteorológicas (Tabla 2). Los aumentos detectados en temperatura promedio anual durante $1960-2015$ fueron de $0.65^{\circ} \mathrm{C}$ (PAL), $0.82{ }^{\circ} \mathrm{C}$ (MAC), $0.56{ }^{\circ} \mathrm{C}$ (PAR), $0.56{ }^{\circ} \mathrm{C}$ (TRE) y $0.34{ }^{\circ} \mathrm{C}(\mathrm{VIL})$, valores estimados con respecto al valor promedio histórico de cada estación meteorológica.

Clima actual. La cantidad estimada de precipitación histórica recibida en la RBPC osciló de 1697 a 2291 $\mathrm{mm}$ anuales, siendo las zonas ubicadas hacia el noroeste y el sur, las que mayor y menor cantidad de precipitación acumulada anual recibieron (Figura 2A). 
Las fluctuaciones en temperatura promedio anual detectadas en 1960-2015 fueron similares (26.67 a $26.88^{\circ} \mathrm{C}$ ) en la ANP (Figura 2B).

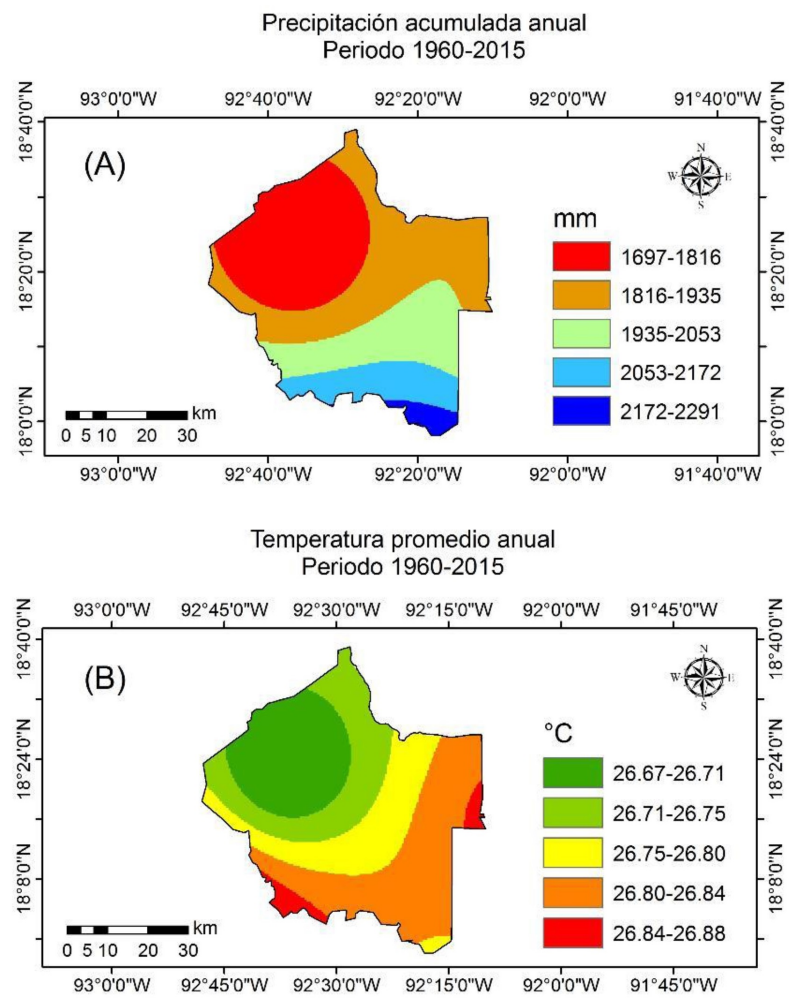

Figura 2: Precipitación acumulada anual $(A)$ y temperatura promedio anual (B) estimados en la RBPC durante 1960-2015.

Clima futuro. Las estimaciones del escenario conservador (MCG CNRMCM5 RCP 4.5) de precipitación proyectaron disminuciones máximas de $616 \mathrm{~mm}$ en la cantidad de precipitación acumulada anual, donde un $91.39 \%$ de la superficie de la RBPC disminuirá de 4 a $371 \mathrm{~mm}$ durante 2045-2069 (Figura 3A). Mientras que, el escenario extremo (MCG HADGEM2_ES RCP 8.5) estimó disminuciones máximas de $678 \mathrm{~mm}$, donde un $88.92 \%$ de la superficie de la RBPC reducirá de 44 a $424 \mathrm{~mm}$ de precipitación anual en el 2045-2069 (Figura 3B). Para la temperatura promedio anual, el escenario conservador (MCG CNRMCM5 $\mathrm{RCP} 4.5$ ) proyectó un incremento máximo de $1.55^{\circ} \mathrm{C}$; donde un $98.65 \%$ de la superficie de la RBPC aumentará entre 0.54 y $1.35^{\circ} \mathrm{C}$ para 2045-2069 (Figura 4A). En el caso del escenario extremo (MCG HADGEM2_
ES RCP 8.5) se calculó un aumento máximo de 3.55 ${ }^{\circ} \mathrm{C}$, donde un $99.18 \%$ de la superficie de la RBPC aumentará de 2.12 a $3.26{ }^{\circ} \mathrm{C}$ para 2045-2069 (Figura 4B).

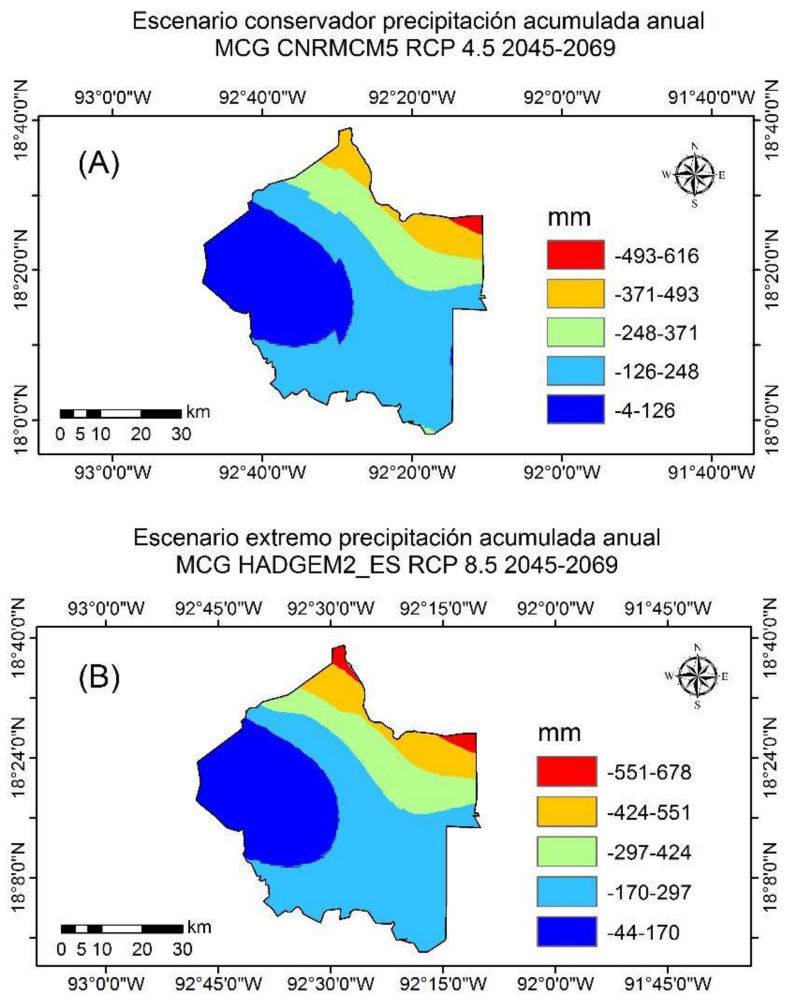

Figura 3: Escenarios de precipitación acumulada anual; conservador (A) y extremo (B) del periodo 2045-2069 en la RBPC.

Las proyecciones a futuro lejano (2075-2099) en la RBPC representan alertas que requieren de acciones inmediatas de adaptación y mitigación al cambio climático, debido a que estima la disminución en la cantidad de la precipitación acumulada anual de hasta $674 \mathrm{~mm}$ por parte del escenario conservador (MCG CNRMCM5 RCP 4.5); donde un $88.85 \%$ de la superficie de RBPC disminuirá la precitación entre 40 y $420 \mathrm{~mm}$ durante 2075-2099 (Figura 5A). Mientras que el escenario extremo (MCG HADGEM2_ES RCP 8.5) proyectó disminuciones máximas de 767 $\mathrm{mm}$; donde en un $98.43 \%$ de la superficie de la RBPC disminuirá la precipitación entre 107 y 503 mm la precipitación hacia finales de siglo (Figura 5B). 


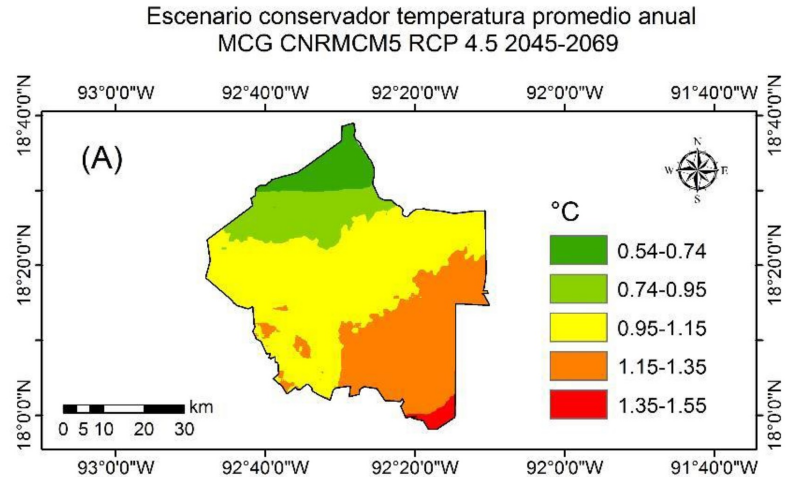

Escenario extremo temperatura promedio anual MCG HADGEM2_ES RCP 8.5 2045-2069

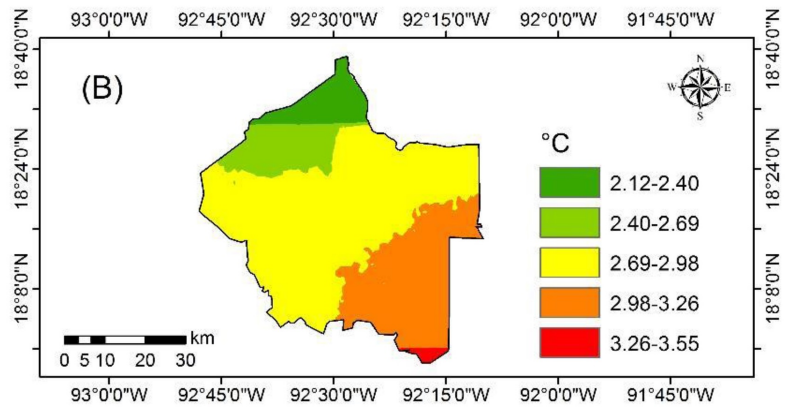

Figura 4: Escenarios de temperatura promedio anual; conservador (A) y extremo (B) del periodo 2045-2069 en la RBPC.

Los valores calculados de temperatura promedio anual señalan que la RBPC sera más cálida a futuro (2045-2069 y 2075-2099). De acuerdo con el escenario climático conservador (MCG CNRMCM5 RCP 4.5) se esperaría que la RBPC aumente su temperatura promedio anual hasta 1.77 ${ }^{\circ} \mathrm{C}$, donde un $98.50 \%$ de la superficie del ANP aumentará entre 0.80 y $1.57{ }^{\circ} \mathrm{C}$ hacia finales de siglo (Figura 6A). Mientras que el escenario extremo (MCG HADGEM2_ES RCP 8.5) estimó un aumento máximo de $5.82{ }^{\circ} \mathrm{C}$; presentando un $99.21 \%$ de la superficie del ANP un aumento entre 3.90 y $5.43{ }^{\circ} \mathrm{C}$ de 2075 2099 (Figura 6B). Este último escenario climático es el más catastrófico que pudiera llegar a presentarse en la RBPC.
Escenario conservador precipitación acumulada anual MCG HADGEM2 ES RCP 4.5 2075-2099

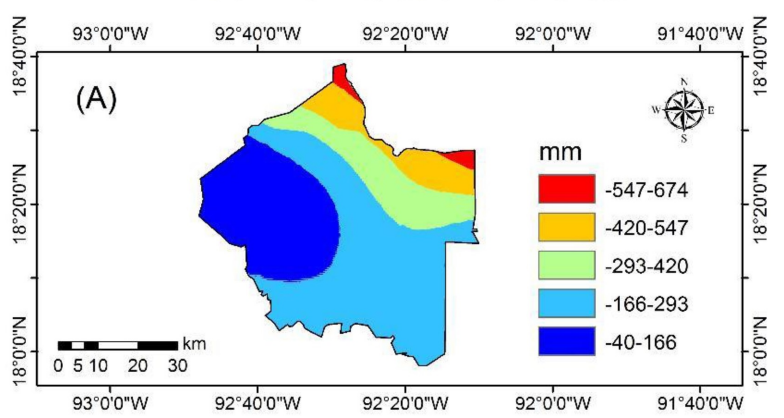

Escenario extremo precipitación acumulada anual MCG HADGEM2_ES RCP 8.5 2075-2099

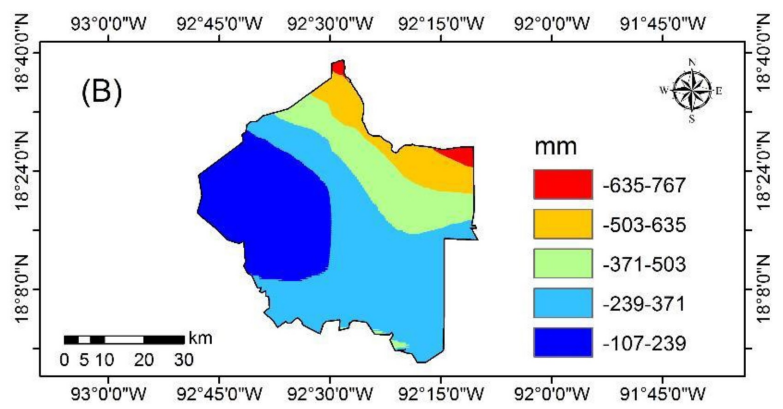

Figura 5: Escenarios de precipitación acumulada anual; conservador (A) y extremo (B) del periodo 2075-2099 en la RBPC.

Afectación por cambio climático. Los escenarios climáticos conservadores (MCG CNRMCM5 RCP 4.5) proyectan una afectación clasificada como baja (aumento en temperatura promedio anual de $0.5 \mathrm{a}$ $1{ }^{\circ} \mathrm{C}$ ) en el $25.70 \%$ de la RBPC y moderada (incremento en temperatura promedio anual de $1 \mathrm{a} 2{ }^{\circ} \mathrm{C}$ ) en el 73\% del ANP durante el periodo 2045-2069 (Figura 7A). Mientras que, hacia futuro lejano se calculó una afectación moderada en el $92.73 \%$ de la superficie de la RBPC (Figura 7B). Asimismo, el incremento en el nivel del mar de 13 a $33 \mathrm{~cm}$ ocasionará la inclusión de agua salada en el 49.3 y $49.5 \%$ de la superficie de la RBPC durante 2045-2069 y 2075-2099, respectivamente (Figura 8) lo que propiciaría en la formación de delta costero en el ANP y un cambio drástico en las comunidades vegetales de agua dulce. 
Escenario conservador temperatura promedio anual MCG CNRMCM5 RCP 4.5 2075-2099

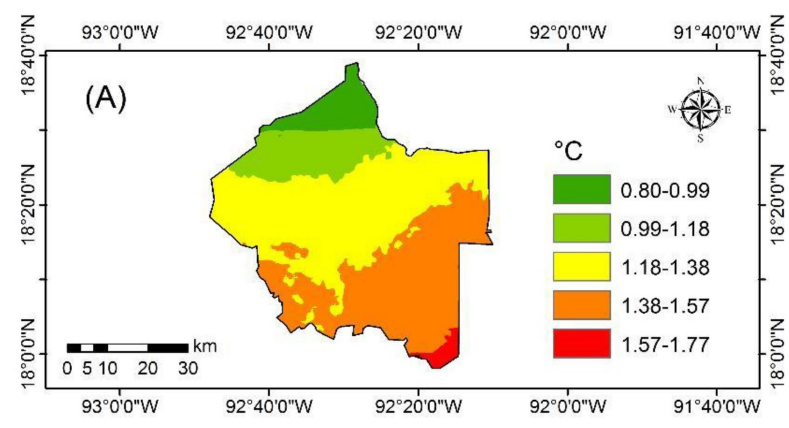

Escenario extremo temperatura promedio anual MCG HADGEM2_ES RCP 8.5 2075-2099

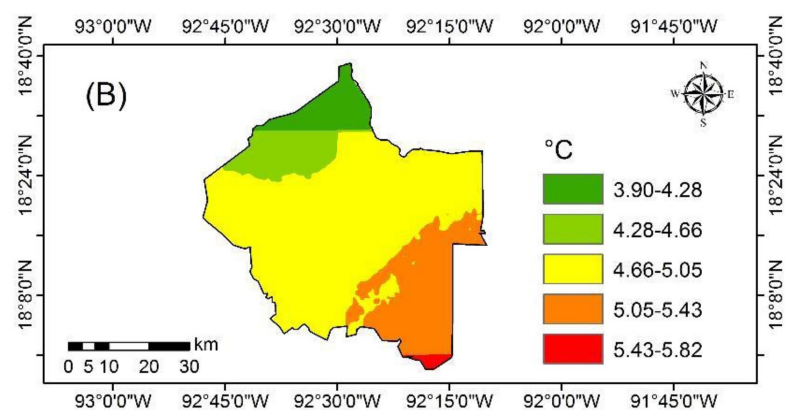

Figura 6: Escenarios de temperatura promedio anual; conservador (A) y extremo (B) del periodo 2075-2099 en la RBPC.

\section{DISCUSIÓN}

Las tendencias similares registradas en los patrones de precipitación acumulada anual de las seis estaciones meteorológicas se deben a la cercanía geográfica con el Golfo de México, lo que ocasiona que reciban mayor precipitación por efectos de la convergencia friccional entre las masas de aire frío y caliente (García 1974, Oerlemans 1980, Gutiérrez et al. 2006). La temporada de sequías registrada durante febrero-abril de 1960-2012 presentó un índice de severidad muy bajo a nulo de 0 a 1.18, lo que indica disponibilidad de humedad (Palmer 1968). La casi nula afectación de las sequías en la RBPC se debe a la alta disponibilidad de humedad en ríos, lagunas y el Golfo de México (Barba et al. 2006), lo que provoca que el efecto de las sequías sea menos intenso y de menor duración en comparación con lo registrado en las regiones centro y norte del país (Stahle et al. 2016, Martínez-Sifuentes et al. 2020).

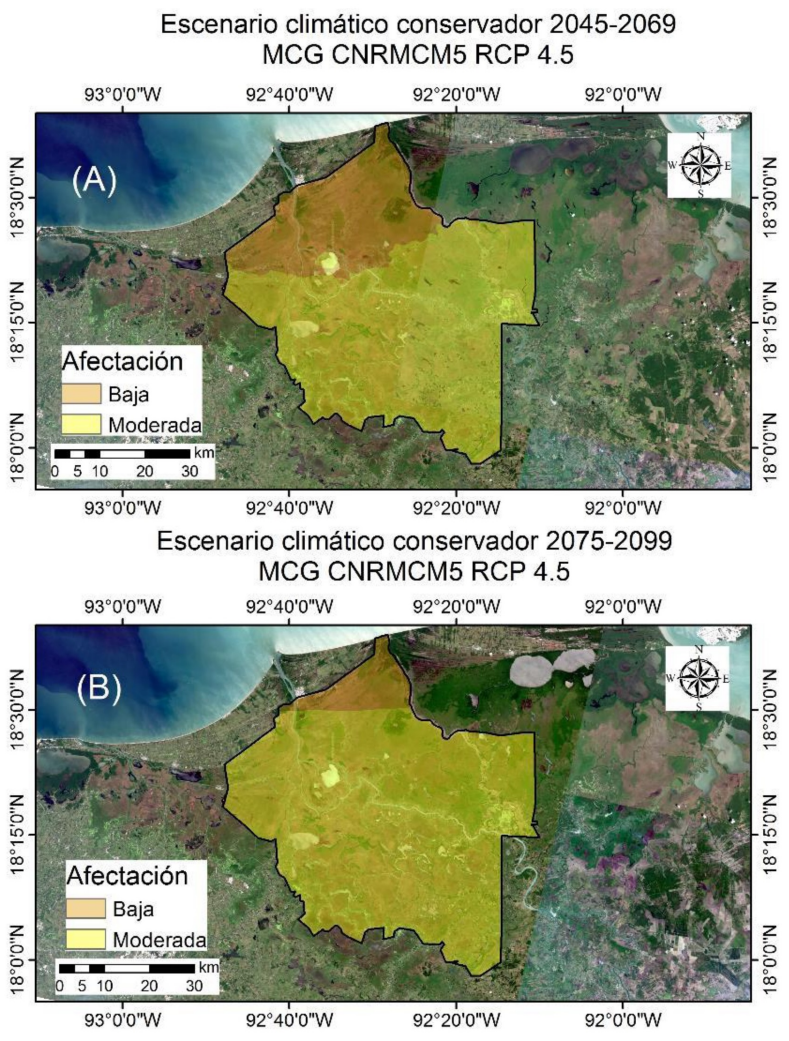

Figura 7: Niveles de afectación por cambio climático durante 20452069 (A) y 2075-2099 (B) en la RBPC.

Los resultados Mann-Kendall indicaron ausencia de tendencias de cambio climático actual (19602015) en los regímenes de precipitación anual acumulada de las seis estaciones meteorológicas analizadas. Tendencias similares han sido reportadas por Bautista et al. (2013) en precipitación acumulada mensual de 1969-2006 en el municipio de Abalá, Yucatán, y por de la Mora et al. (2016) en los patrones de precipitación anual de 16 estaciones meteorológicas localizadas en el estado de Chiapas durante 1960-2009, lo que indica que las tendencias de precipitación en el país suele ser erráticos (CONAFOR 2013, CONANP 2015).

La temperatura promedio anual de 1960-2015 presentó nula estacionalidad en las series de tiempo de las seis estaciones evaluadas, lo que concuerda con el tipo de clima presente en las zonas tropicales del sureste Mexicano (Bautista et al. 2013, Manzanilla y Aguirre 2017) donde se registraron 


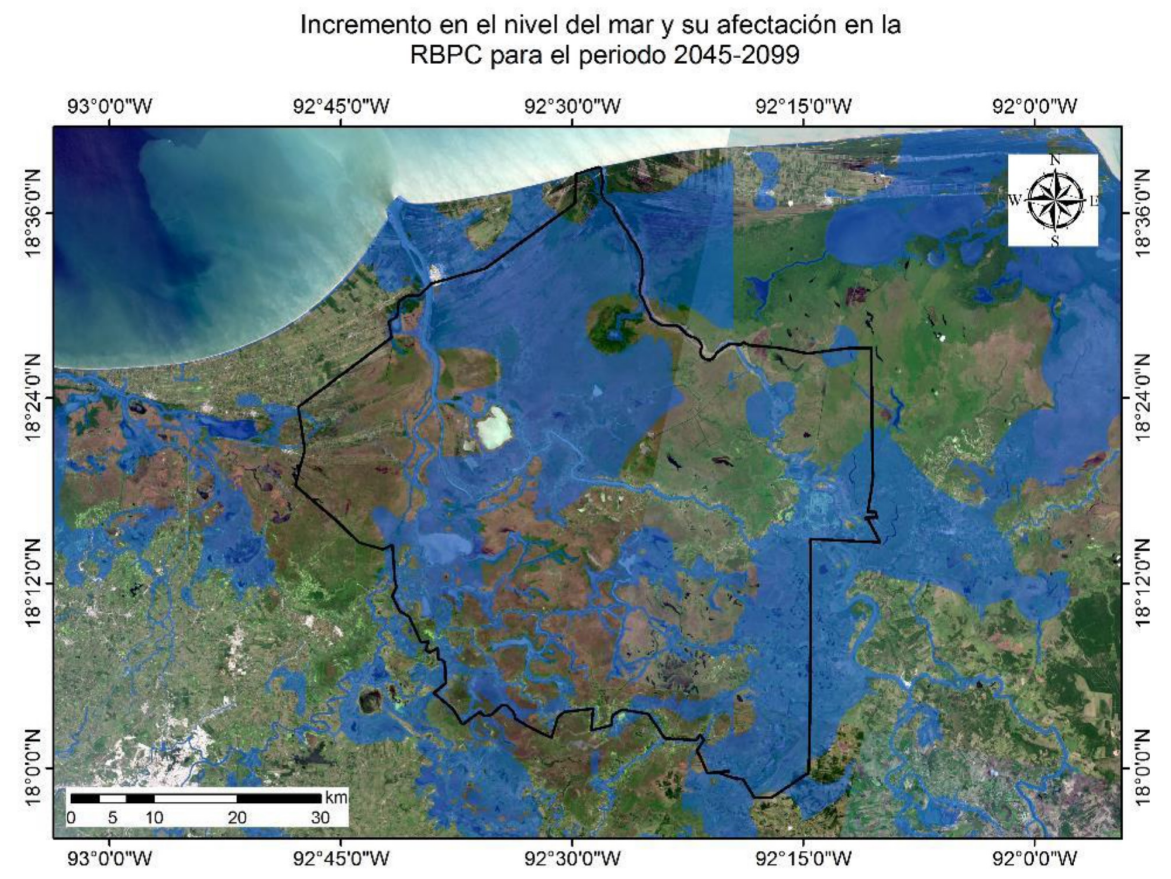

Figura 8. Inclusión de 13 a 33 cm de agua marina en la RBPC durante el periodo 2045-2099.

temperaturas constantes durante todo el año y la estacionalidad es casi nula. La presencia de tendencias significativas $(p<0.05)$ de aumento en temperatura de cinco estaciones indicaron que la temperatura promedio anual de la RBPC ha aumentado significativamente durante 1960-2015. Resultados similares de incremento en temperatura máxima anual han sido reportados por de la Mora et al. (2016) en 10 de 16 estaciones meteorológicas en el estado de Chiapas durante 1960-2009, lo que indicó que las zonas tropicales del sureste mexicano se están haciendo más cálidas (Peralta-Hernández et al. 2009, Manzanilla y Aguirre 2017). Por otra parte, Bautista et al. (2013) hallaron una tendencia significativa de enfriamiento en la temperatura máxima, promedio y mínima anual en el municipio de Abalá, Yucatán, lo cual difiere con los resultados de este estudio. Este tipo de tendencias de disminución en temperatura son raras y contrarias a lo que comúnmente se esperaría de un análisis climático en sitios con condiciones climáticas similares. Lo que lleva a pensar sobre la existencia de modificaciones en el clima local, producidas por los efectos dinámicos del cambio climático, los cuales afectan de manera heterogénea a cada región geográfica del planeta.

Escala espacial. El número relativamente pequeño de estaciones meteorológicas empleadas en la generación de las capas climáticas se debe a la limitación en extensión, duración y de calidad de los registros meteorológicos disponibles en la zona de estudio, siendo esta limitación en datos climáticos una constante en México (Cuervo-Robayo et al. 2014). Por ello se deben mejorar y fortalecer las redes de monitoreo climático en el sureste de México. A pesar de lo anterior, las interpolaciones (IDW) generadas se consideran robustas, confiables y representativas de la RBPC, la cual tiene escasa variabilidad en altitud, lo que permite aminorar los sesgos derivados por los efectos orográficos del sitio de estudio.

Es ampliamente aceptado por la comunidad científica que la modelación bajo escenarios de cambio climático posee un cierto grado de incertidumbre inherente no determinística. Debido a la incertidumbre de los escenarios de cambio climático, 
se probaron y analizaron varios MCG (CNRMCM5 y HADGEM2_ES) y RCP (4.5 y 8.5) para los horizontes temporales 2045-2069 y 2075-2099 en la RBPC, lo cual es ampliamente recomendado por varios autores en México (Fernández-Eguiarte et al. 2015, Manzanilla et al. 2018). De tal manera que se obtuvieron diversos panoramas de valores mínimos y máximos de las variables climáticas analizadas. Asimismo, se sabe con certeza que las tendencias globales de cambio climático señalan escenarios más cálidos y secos a futuro, los cuales se derivan del aumento en las emisiones de gases de efecto invernadero. Pero en escalas regionales y locales, otros factores (microclimáticos y geográficos) pudieran estar presentes (Cuervo-Robayo et al. 2014, Taibi et al. 2019), generando tendencias de tipo: calentamiento (mundial), enfriamiento (regional y local) y sin cambios notorios (Bautista et al. 2013, IPCC 2014, Fernández-Eguiarte et al. 2015).

La reducción de la resolución espacial para estimar superficies climáticas de escala regional (1 $\mathrm{km}^{2}$ ) a local se ha empleado con anterioridad en las ANPs Nevado de Colima, Nevado de Toluca y Pico de Orizaba en el centro de México por Manzanilla et al. (2018), dicho reajuste se utilizan para mejorar los resultados en estimaciones de superficies climáticas de áreas relativamente pequeñas. Por lo que su aplicación es recomendable.

Escenarios climáticos. Los escenarios de cambio climático generados en México en el siglo XXI, proyectaron un aumento en temperatura promedio anual de $2.3{ }^{\circ} \mathrm{C}$ hacia 2060 y de $3.7^{\circ} \mathrm{C}$ en 2090 (Sáenz-Romero et al. 2010). Estos valores son similares a los proyectados por los modelos conservadores (RCP 4.5). Sin embargo, los escenarios extremos (RCP 8.5) estiman un aumento en temperatura promedio anual de entre 2.73 y $3.55{ }^{\circ} \mathrm{C}$ para 2045-2069 (Figura 5B) y de 3.42 a $5.82{ }^{\circ} \mathrm{C}$ para 2075-2099 (Figura 7B). Escenarios extremos (MCG HADGEM2_ES) generados en el complejo Pantanos de Centla-Laguna de Términos por CONANP y PNUD México (2019) determinaron un aumento en temperatura promedio anual $\left(6.6^{\circ} \mathrm{C}\right)$ mayores a los obtenidos en este estudio hacia finales de siglo.

De acuerdo con la Organización de la Naciones Unidas para la Alimentación y la Agricultura (FAO 2003), debido a los efectos del calentamiento global, en el 2050 alrededor de $25 \%$ de la biodiversidad mundial afrontaría un mayor riesgo de extinción. Aunado a este escenario, el IPCC (2014) estableció que un aumento de 1 a $2{ }^{\circ} \mathrm{C}$ en la temperatura promedio anual representaría un daño moderado a la biodiversidad global, mientras que un incremento mayor de $3.5^{\circ} \mathrm{C}$ resultaría perjudicial para la mayoría de los ecosistemas terrestres y marinos, lo que ocasionará extinciones de entre 40 y $70 \%$ de las especies del mundo (IPCC 2007). Estos últimos escenarios representan el panorama climático no deseado a manifestarse en la RBPC.

Para la precipitación, se presentan tendencias irregulares a nivel mundial (IPCC 2014) y en México (CONAFOR 2013, CONANP 2015). Los escenarios realizados en México sugieren una disminución promedio en la precipitación anual acumulada de entre 5 y $17 \%$ para 2050, y de hasta $28 \%$ hacia finales de siglo (Sáenz-Romero et al. 2010, Magaña et al. 2012, Manzanilla et al. 2018). Resultados que son similares a los determinados en este estudio en disminución (8.5 y 19.7\%) de precipitación acumulada anual en la RBPC por los modelos conservadores para 2045-2069 y 2075-2099. Al respecto, Sáenz-Romero et al. (2010) y Magaña et al. (2012) reportan que las regiones del norte de México serían las zonas que mayores modificaciones presentarían en sus regímenes de temperatura y precipitación a partir del 2050, las cuales afectarían a los ecosistemas forestales, principalmente los bosques de coníferas (Sáenz-Romero et al. 2015, Cruz-Cárdenas et al. 2016, Manzanilla-Quiñones et al. 2019).

Los escenarios climáticos generados en las regiones tropicales del sureste de México estiman que los bosques secos tenderían a expandir sus áreas de distribución un 7.4\% (Villers y Trejo 1998); de manera que sitios como la Reserva de la Biosfera Selva el Ocote (REBISO) en Chiapas se volverían más cálidos (Manzanilla y Aguirre 2017). Mientras que la RBPC también sería más cálida pero con la variante de que presentaría serios problemas ecológicos, bio- 
geoquímicos en el agua y de erosión en la franja costera por la inclusión de agua salada (IPCC 2014, Ramos et al. 2016, Cruz-Ramírez et al. 2019).

El aumento en el nivel del mar y en temperatura promedio anual propiciará desequilibrio en los ciclos biogeoquímicos del agua (hípersalinidad), ocasionando que las poblaciones de peces dulceacuícolas tengan una menor tolerancia térmica, crecimiento, metabolismo y bajo éxito reproductivo (Fry 1971, Cruz-Ramírez et al. 2019), a su vez la inclusión de agua salada traerá un mayor intercambio de especies marinas hacia los humedales (Chávez-López y Rocha-Ramírez 2020) en el 49.3\% de la RBPC a partir de 2045-2069.

En cuanto a los sitios terrestres de alta biodiversidad llamados hotspot dentro de la RBPC, estos podrían dejar de ser un refugio para mamíferos carnívoros de gran talla como el Jaguar (Panthera onca), especies en vías de extinción como el Manatí (Trichechus manatus) y los primates ( $A$. geoffroyi, Alouatta palliata y A. pigra) (INEC 2000, CONANP y PNUD México 2019). El ascenso del nivel del mar propiciará que los ecosistemas se fragmenten y modifiquen su diversidad biológica y ecológica (Molina-Sánchez et al. 2019) más que en la actualidad. Con la consecuente pérdida o disminución de especies clave en la conservación (Jaguar, Manatí y los primates) y de ecosistemas de importancia ecológica como manglar, selva baja espinosa y selva mediana, los cuales podrían extinguirse localmente (Sáenz-Romero et al. 2010, Manterola et al. 2011, Gutiérrez y Trejo 2014, IPCC 2014).

\section{CONCLUSIONES}

Se acepta la hipótesis de que la RBPC se convertiría en un sitio altamente expuesto al cambio climático y cuyos efectos ocasionaran calentamiento y aumento en el nivel del mar. Las proyecciones a futuro prevén un ANP más cálida e inundable por la entrada de agua salada, derivada del incremento del nivel del mar. Por lo que las actividades pendientes a realizar en investigaciones posteriores deben enfocarse sobre cuestiones de manejo integral de la zona costera, control de erosión de la franja costera, estudios ecofisiológicos (capacidad de resiliencia) de especies de invertebrados acuáticos y peces dulceacuícolas, de interacciones entre especies marinas-agua dulce y de conectividad ecológica entre taxones claves en la conservación como el jaguar, Manatí y los primates en las islas de vegetación. Se sugiere realizar acciones de manejo que prioricen la mitigación y adaptación al cambio climático, como la restauración de las zonas terrestres-riparias, incentivar y fomentar los sistemas agroecológicos y sociales e implementar campañas de concientización sobre los efectos del cambio climático y sus implicaciones tanto dentro como fuera del área natural protegida.

\section{AGRADECIMIENTOS}

Al Consejo Nacional de Ciencia y Tecnología (CONACyT) por el apoyo de beca de posgrado número 442987 otorgada al primer autor. A la Facultad de Ciencias Forestales de la Universidad Autónoma de Nuevo León. Al Dr. Cristian Martínez, por su apoyo en la revisión y corrección del Abstract.

\section{LITERATURA CITADA}

Álvarez IP (2013) Corredor Biológico Mesoamericano en México. Biodiversitas 110: 1-5.

Barba E (2005) Valor del hábitat: Distribución de peces en humedales de Tabasco. ECOfronteras 25: 9-11.

Barba E, Rangel MJ, Reyes R (2006) Clasificación de los humedales de Tabasco mediante sistemas de información geográfica. Universidad y Ciencia 22: 101-110.

Bautista F, Bautista-Hernández DA, Álvarez O, Anaya-Romero M, de la Rosa D (2013) Software para identificar las tendencias de cambio climático a nivel local. Un estudio de caso en Yucatán, México. Revista Chapingo 
Serie Ciencias Forestales y del Ambiente 19: 81-90.

Bartlett MS (1937) Propiedades de suficiencia y pruebas estadísticas. Medidas de la Sociedad Estadística Real. Serie unos 160: 268-282.

Chávez-López R, Rocha-Ramírez A (2020) Composición de la comunidad de peces en el estuario ciego laguna El Llano, Veracruz, México. Revista Mexicana de Biodiversidad 91: 1-11.

CITMA (2012) Informe de Cuba a la Conferencia de las Naciones Unidas sobre Desarrollo Sostenible Río+20. Ministerio de Ciencia, Tecnología y Medio Ambiente. Agencia de Medio Ambiente. La Habana, Cuba. 21p.

CLICOM (2020) Registros climáticos disponibles para toda la República Mexicana. Valores anuales y mensuales de precipitación y temperatura. Climatic Computing Project. http://clicom-mex.cicese.mx/. Fecha de consulta: 20 de abril de 2020.

CONANP (2015) Estrategia de cambio climático desde las Áreas Naturales Protegidas: Una convocatoria para la resiliencia de México (2015-2020) 1a edición. Comisión Nacional de Áreas Naturales Protegidas. Secretaría de Medio Ambiente y Recursos Naturales. México. 60p.

CONANP y PNUD México (2019) Resumen ejecutivo del Programa de Adaptación al Cambio Climático del Complejo de las ANP Reserva de la Biosfera Pantanos de Centla-Área de Protección de Flora y Fauna Laguna de Términos. Comisión Nacional de Áreas Naturales Protegidas y Programa de las Naciones Unidas para el Desarrollo. Ciudad de México, México. 20p.

CONAFOR (2013) Bosques, cambio climático y REDD+ en México. Guía básica. $2^{\mathrm{a}}$ edición. Comisión Nacional Forestal. Zapopan, Jalisco, México. 88p.

Cruz-Cárdenas G, López-Mata L, Silva JT, Bernal-Santana N, Estrada-Godoy F, López-Sandoval JA (2016) Potential distribution model of Pinaceae species under climate change scenarios in Michoacán. Revista Chapingo Serie Ciencias Forestales y del Ambiente 22: 135-148.

Cruz-Ramírez AK, Salcedo MA, Sánchez AJ, Barba ME, Mendoza PJD (2019) Relationship among physicochemical conditions, chlorophylla concentration, and water level in a tropical river-floodplain system. International Journal of Environmental Science and Technology 16: 3869-3876.

Cuervo-Robayo AP, Téllez-Valdés O, Gómez-Albores MA, Venegas-Barrera CS, Manjarrez J, Martínez-Meyer E (2014) An update of high-resolution monthly climate surfaces for Mexico. International Journal of Climatology 34: 2427-2437.

de la Mora OC, Ruíz CJA, Flores LHE, Zarazúa VP, Ramírez OG, Medina GG, Rodríguez MVM, Chávez DAA (2016) Índices de cambio climático en el estado de Chiapas, México, en el periodo 1960-2009. Revista Mexicana de Ciencias Agrícolas 13: 2523-2534.

ESRI (2014) ArcGis Desktop: 10.3. Software diseñado para análisis espacial y Sistemas de Información Geográfica. Environmental Systems Research Institute. Redlands, California, U.S.A.

Fry FEJ (1971) The effect of environmental factors on the physiology of fish. In: Hoar WS, Randall DJ (eds). Environmental Relations and Behavior. Fish Physiology Vol. 6. Academic Press. New York, USA. 98p.

García E (1974) Distribución de la precipitación en la República Mexicana. Investigaciones Geográficas 5: 7-20.

García E (1998) Comisión Nacional para el Conocimiento y Uso de la Biodiversidad (CONABIO). Climas (Clasificación de Köppen, modificado por García). Escala 1:1000000. México, D .F., México. Archivo vectorial Shapefile: Tipos de climas. http://www.conabio.gob.mx/informacion/metadata/gis/clima1mgw.xml?_http cache=yes\&_xsl=/db/metadata/xsl/fgdc_html.xsl\&_indent=no. Fecha de consulta: 01 de abril de 2020. 
Gutiérrez E, Trejo I (2014) Efecto del cambio climático en la distribución potencial de cinco especies arbóreas de bosque templado de México. Revista Mexicana de Biodiversidad 85: 179-188.

Gutiérrez MFJ, Varona-Cordero F, Contreras EJ (2006) Caracterización estacional de las condiciones físicoquímicas y de productividad primaria fitoplanctónica de dos lagunas costeras tropicales del estado de Chiapas, México. Hidrobiología 16: 137-146.

INEC (2000) Programa de manejo Reserva de la Biosfera Pantanos de Centla. Carabias LJ, Provencio E, de la Maza EJ, Romero GJC (ed). Instituto Nacional de Ecología. Primera edición. México. 222p.

INEGI (2014) Conjunto de datos vectoriales edafológicos. Continuo Nacional. Escala 1:250 000. Serie II. Instituto Nacional de Estadística, Geografía e Informática. Aguascalientes, Aguascalientes, México. Archivo vectorial Shapefile: Tipos de suelos. http://www.conabio.gob.mx/informacion/metadata/gis/eda250s2gw.xml? httpcache=yes\&_xsl=/db/metadata/xsl/fgdc_html.xsl\&_indent=no. Fecha de consulta: 01 de abril de 2020.

INEGI (2016) Uso del suelo y vegetación. Escala 1:250 000.Serie VI (capa unión). Instituto Nacional de Estadística, Geografía e Informática. Aguascalientes, Aguascalientes, México. Archivo vectorial Shapefile: Uso del suelo y vegetación. http://www.conabio.gob.mx/informacion/metadata/gis/usv250s6gw.xml?_httpcache=yes \&_xsl=/db/metadata/xsl/fgdc_html.xsl\&_indent=no. Fecha de consulta: 05 de abril de 2020.

INEGI (2020) Continuo Mexicano de Elevaciones para Tabasco y Campeche. Escala $15 \mathrm{~m}$. Instituto Nacional de Estadística, Geografía e Informática. Aguascalientes, Aguascalientes, México. Archivo ráster: modelos digitales de elevaciones para Tabasco y Campeche. https://www.inegi.org.mx/app/geo2/elevacionesmex/. Fecha de consulta: 10 de abril de 2020.

IPCC (2007) Climate change 2007: Synthesis report. In: Pachauri RK, Reisinger A (ed) Contribution of Working Groups I, II and III to the fourth assessment report of the Intergovernmental Panel on Climate Change. Panel Intergubernamental de expertos sobre el cambio climático. Geneva, Switzerland. 104p.

IPCC (2013) Resumen para responsables de políticas. En: Stocker TF, Qin D, Plattner GK, Tignor M, Allen SK, Boschung J, Nauels A, Xia Y, Bex V, Midgley PM (ed) Cambio climático 2013: Bases físicas. contribución del Grupo de trabajo I al V Informe de Evaluación del Grupo Intergubernamental de Expertos sobre el cambio climático. Climate Change 2013. Panel Intergubernamental de expertos sobre el cambio climático. Ginebra, Suiza. 27p.

IPCC (2014) Resumen para responsables de políticas. Contribución del grupo de trabajo II al Quinto Informe de Evaluación del Panel Intergubernamental de Expertos sobre el Cambio Climático. En Field CB, Barros VR, Dokken DJ, Mach KJ, Mastrandrea MD, Billir TE, Chatterjee M, Ebi KL, Estrada YO, Genova RC, Girma B, Kissel ES, Levy AN, MacCracken S, Mastrandrea PR, White LL (ed) Cambio climático 2014: Impactos, adaptación y vulnerabilidad. Panel Intergubernamental de Expertos sobre Cambio Climático. Ginebra, Suiza. 34p.

Fernández-Eguiarte A, Zavala-Hidalgo J, Romero-Centeno R, Conde-Álvarez AC, Trejo-Vázquez RI (2015) Actualización de los escenarios de cambio climático para estudios de impacto, vulnerabilidad y adaptación en México y Centroamérica. Universidad Nacional Autónoma de México, Instituto Nacional de Ecología y Cambio Climático. México. 22p.

Fry FEJ (1971) The effect of environmental factors on the physiology of fish. In: Hoar WS, Randall DJ (ed) Environmental Relations and Behavior. Fish Physiology Vol. 6. Academic Press. New York, USA. 98p.

Jáuregui OE (2003) Algunos conceptos modernos sobre la circulación general de la atmósfera. Investigaciones Geográficas 50: 121-143. 
Kendall MG (1938) A new measure of rank correlation. Biometrika 30: 81-93.

López-Jiménez LN, Jiménez- López DA, Castillo-Acosta O, Gallardo-Cruz JA, Fernández-Montes de Oca AI (2020) Plantas vasculares de la Reserva de la Biosfera Pantanos de Centla, México. Botanical Sciences 98: 159-204.

Magaña V, Zermeño D, Neri C (2012) Climate change scenarios and potential impacts on water availability in northern Mexico. Climate Research 51: 171-184.

Manterola C, Conde AD, Colchero F, Rivera A, Huerta E, Soler A, Pallares E (2011) El jaguar como elemento estratégico para la conservación. En Becerra R, Vargas LE, Ramírez B, Solís V (ed.) Corredor Biológico Mesoamericano México. Comisión Nacional para el Conocimiento y Uso de la Biodiversidad. México. pp: $1-126$

Manzanilla QU, Aguirre COA (2017) Zonificación climática actual y escenarios de cambio climático para la Reserva de la Biosfera Selva el Ocote en Chiapas, México. En: Ruíz ML, Álvarez GG, Ramírez N, Cruz BS (ed) Vulnerabilidad social y biológica ante el cambio climático en la Reserva de la Biosfera Selva El Ocote. ECOSUR. San Cristóbal de las Casas, Chiapas, México. pp: 25-66.

Manzanilla QU, Aguirre COA, Jiménez PJ, Treviño GEJ, Yerena YJI (2018) Escenarios de cambio climático (CMIP-5) para tres áreas naturales protegidas en el Eje Neovolcánico Transversal. Revista Mexicana de Ciencias Forestales 9: 515-537.

Manzanilla-Quiñones U, Aguirre-Calderón OA, Jiménez-Pérez J, Treviño-Garza EJ, Yerena-Yamallel JI (2019) Distribución actual y futura del bosque subalpino de Pinus hartwegii Lindl en el Eje Neovolcánico Transversal. Madera y Bosques 25(2): e2521804. doi: 10.21829/myb.2019.2521804.

Manzanilla-Quiñones U, Aguirre-Calderón OA, Jiménez-Pérez J, Villanueva-Díaz J (2020) Sensibilidad climática en anchuras de anillos de crecimiento de Pinus hartwegii: Una especie alpina mexicana con potencial dendroclimático. Revista Mexicana de Biodiversidad 91(2020): e913117. doi: https://doi.org/10.22201/ib.20078 706e.2020.91.3117.

Martínez-Sifuentes AR, Villanueva-Díaz J, Estrada-Ávalos J (2020) Runnoff reconstruction and Climatic influence with tree rings, in the Mayo river basin, Sonora, Mexico. iforest 13: 98-106.

McCuen RH, Snyder WM (1986) Hydrological modelling: statistical methods and applications. Prentice-Hall. Englewood Cliffs, New Jersey, USA. 568p.

Miranzo M, del Río C (2015) Las consecuencias del cambio climático en el Magreb. Revista UNISCI Discussion Papers 39: 127-150.

Molina SA, Delgado VP, González-Rodríguez A, González C, Gómez-Tagle RAF, López-Toledo L (2019) Spatiotemporal approach for identification of critical conservation areas: a case study with two pine species from a threatened temperature forest in Mexico. Biodiversity and Conservation 28: 1863-1883.

Oerlemans J (1980) A case of a Subsynoptic Disturbance in a Polar Outbreak. Quartely Journal of the Royal Meteorological Society 106: 313-325.

Palmer WC (1968) Keeping track of crop moisture conditions nationwide: the new Crop Moisture Index. Weatherwise 21: 156-161.

Peralta-Hernández AR, Balling RC, Barba-Martínez LR (2009) Analysis of near-surface diurnal temperature variations and trends in southern Mexico. International Journal of Climatology 29: 205-209.

QGIS (2018) QGIS Versión 3.2. Sistema de Información Geográfica libre y de código abierto. Disponible en https://qgis.org/es/site/. Fecha de consulta: 15 de abril de 2020. 
R Core Team (2020) R: a language and environment for statistical computing. R Foundation for Statistical Computing. Viena, Austria. Versión 4.0. htpp://www.r-project.org. Fecha de consulta: 30 de abril de 2020.

Ramos RR, Gama CLM, Núñez GJC, Sánchez HR, Hernández TH, Álvarez RO (2016) Adaptación del modelo de vulnerabilidad costera en el litoral tabasqueño ante el cambio climático. Revista Mexicana de Ciencias Agrícolas 13 (Pub. Núm. Esp.): 2551-2563.

Sáenz-Romero C, Rehfeldt GE, Crookston NL, Pierre D, St-Amant R, Beaulieu J, Richardson B (2010) Contemporary and projected Spline Climate surfaces for Mexico and their use in understanding Climate-plant relationships. Climatic Change 102: 595-623.

Sáenz-Romero C, Rehfeldt GE, Ortega-Rodríguez JM, Marin-Togo MC, Madrigal-Sánchez X (2015) Pinus leiophylla suitable habitat for 1961-1990 and future climate. Botanical Sciences 93: 709-718.

Schulz EF (1976) Problems in applied hydrology. In: Water Resources Publications (ed.) Elementary statistical properties. Fort Collins. Water Resources Publications. pp: 31-73

SEMARNAP (1997) Primera comunicación nacional ante la convención marco de las Naciones Unidas. Secretaría de Medio Ambiente, Recursos Naturales y Pesca. México. 139p.

Soria-Barreto M, González-Díaz AA, Castillo-Domínguez A, Álvarez-Pliego N, Rodiles-Hernández R (2018) Diversidad íctica en la cuenca del Usumacinta, México. Revista Mexicana de Biodiversidad 89 (Suplemento 2018): S100-S117.

Stahle DW, Cook ER, Burnette D, Villanueva DJ, Cerano PJ, Burns J, Griffin D, Howard JM (2016) The mexican drought atlas: Tree-ring reconstructions of the soil moisture balance during the late pre-Hispanic, colonial, and modern eras. Quaternary Science Reviews 149: 34-60.

Taibi S, Meddi M, Mahé G (2019) Seasonal rainfall variability in the souther Mediterranea border: Observations, regional model simulations and future climate projections. Atmósfera 32: 39-54.

Trinidad-Ocaña C, Juárez J, Sánchez AJ, Barba E (2018) Diversidad de moluscos y crustáceos acuáticos en tres zonas en la cuenca del río Usumacinta, México. Revista Mexicana de Biodiversidad 89 Suplemento: S65-S78.

Velasco C, Sánchez AJ, Florido R (2005) New locality record of Uhlorchestia uhleri (Peracarida: Amphipoda: Talitridae) in the coastal zone of the southern Gulf of Mexico. Crustaceana 78: 499-502.

Vega-Moro A (2005) Plan de conservación para la Reserva de la Biosfera Pantanos de Centla y el Área de Protección de Flora y Fauna de Laguna de Términos. Pronatura, The Nature Conservancy, Comisión Nacional de áreas Naturales Protegidas. Ciudad de México, México. 232p.

Villers RL, Trejo VRI (1998) Climate change on Mexican forests and natural protected areas. Global Environmental Change 8: 141-157. 